\title{
Superatoms and quasimolecules in quasi-zero-dimensional nanoheterostructures: Future research
}

\author{
Sergey I. Pokutnyi, Petr P. Gorbyk \\ Chuiko Institute of Surface Chemistry of National Academy of Sciences of Ukraine, Kyiv, Ukraine \\ Email address: \\ pokutnyi_sergey@inbox.ru (S. I. Pokutnyi)
}

\section{To cite this article:}

Sergey I. Pokutnyi, Petr P. Gorbyk. Superatoms and Quasimolecules in Quasi-Zero-Dimensional Nanoheterostructures: Future Research. Optics. Special Issue: Optics and Spectroscopy of the Charge Carriers and Excitons States in Quasi - Zero - Dimensional Nanostructures. Vol. 3, No. 6-1, 2014, pp. 42-47. doi: 10.11648/j.optics.s.2014030601.16

\begin{abstract}
This review discusses the current state of research of artificial atoms (superatoms) (quasiatomic nanoheterostructures) and more complex nanostructures based on them - synthetic molecules, proposed a new model of an artificial atom, satisfactorily explaining its electronic properties, as well as the prospects for the development of the new scientific field.
\end{abstract}

Keywords: Superatoms (Artificial Atoms), Quasimolecules (Artificial Molecules), Nanoheterostructures, Quantum Dots, Electron, Hole, Energy Spectrum

\section{Introduction}

For the development of nanophysics and nanochemistry fruitful is the idea of "artificial atom" (or superatom) [1-9]. Superatom (quasiatomic nanoheterostructures) consists of a core (quantum dots (QDs)) of radius $a$ with dielectric permittivity (DP) $\varepsilon_{2}$, which is contained in the volume of the semiconductor (or dielectric) material; core is surrounded by a dielectric (semiconductor) sensor with DP $\varepsilon_{1}$ [2- 9]. The term "artificial atom" (superatom) is legitimate, given the discrete the nature of the spectra of the electronic states superatomic and natural atoms [2-9], as well as the similarity of their chemical activity [10].

Quasi-zero-dimensional nanosystems (QN) research consisting of nanocrystals of spherical shape - so-called QDs with radii $a=1-10 \mathrm{~nm}$, containing within its scope semiconductors (CdS, CdSe, GaAs, Ge, Si, ZnSe) and insulators (A12O3), grown in dielectric (semiconductor) matrices, has received increased attention due to their unique photoluminescence properties, the ability to efficiently emit light in the visible or near-infrared bands at room temperature [11-21]. Optical and electro-optical properties of QN (which in some cases exhibit the properties superatomic) largely determines the energy spectrum of space - bounded quasiparticles (electrons, holes, excitons, biexcitons, phonons, polarons, positrons, positrons, etc.) [2 -9].

Arising in heterogeneous structures of heterogeneity (which can be considered as nanoparticles and nanoclusters), due, in particular, fluctuations in dopant concentration and chemical composition, synthesis conditions in multicomponent systems, plays a special role in nanophysical and nanochemical superatomic formation processes [1-4]. Localization of charge carriers over the surfaces of such irregularities under certain conditions leads to the formation of artificial atoms [2-9]. The formation of such superatomic accompanied by the emergence of the electronic spectra of QN acceptor levels, the nature of which is unknown in most cases, as well as a sharp drop in the mobility of the charge carriers in QN [13 21]. For example, in experimental studies [18,19] is set acceptor character nanoparticles Agm (AgmS)n in photoemulsions.

This review deals with the existing theoretical approaches to the description of artificial atoms and more complex nanostructures based on them - synthetic molecules, the proposed new model of artificial atoms, satisfactorily explaining its electronic properties, as well as the prospects for the development of appropriate new research direction. 


\section{Artificial Atoms and Molecules in Nanoheterostructures}

In the study of the optical properties of glass samples with QDs cadmium sulfide and zinc selenide in the experimental study [13] found that the electron can be localized on the surface of the QD, and the hole at the same time moving in the amount of QD. In [14,15] studied experimentally the QN consisting of QDs alumina grown in dielectric matrices. Since the values of band gaps of dielectric matrices (about $3.3 \mathrm{eV}$ ) is smaller than the band gap of alumina QD $(7.2 \mathrm{eV})$, there is a possibility of an electron from runoff volume QD in the matrix and the localization of the electron in the potential well above the QD (a hole at it moves in the volume QD). Thus, in the [1315], apparently for the first time established experimentally appearance of artificial atoms as nuclei containing QDs cadmium sulfide, zinc selenide and the alumina placed in a dielectric matrix.

In [2-9] proposed a new model of an artificial atom (superatom), which is a QN, consisting of a spherical QD (core superatom) of radius $a$, which contains in its volume semiconductor (dielectric) with $\operatorname{PD} \varepsilon_{z}$, surrounded by a dielectric matrix with $\mathrm{PD} \varepsilon_{1}$ (relative $\mathrm{DP} \varepsilon=\left(\varepsilon_{2} / \varepsilon_{1}\right) \gg 1$ ). In a volume QD moves hole $h$ with the effective mass $m_{h}$, and electron $e$ from the effective mass $m_{6}{ }^{(1)}$ is in a dielectric matrix. In such QN lowest electronic level is located in the matrix, and the most humble level of the hole is in the amount of QD. Large shift of the valence band (about 700 $\mathrm{meV}$ ) is the localization of holes in the volume QD. Large shift of the conduction band (about $400 \mathrm{meV}$ ) is a potential barrier for electrons (electrons move in the matrix and do not penetrate the volume QD) [13-15]. Energy of the Coulomb interaction of the electron with the hole, as well as the energy of the polarization of electron interaction with the interface (QD - matrix) (as DP $\varepsilon_{2}$ QD is far superior to the DP matrix $\varepsilon_{1}$ ) cause the localization of the electron in the potential well above the QD [5-9].

In [5-9], in the framework of the modified method of the effective mass [22], developed a theory superatom of spatially separated electrons and holes (the hole is in the amount of QD, and the electron is localized on the spherical surface interface (QD - matrix)). As a core advocate QDs containing in its volume semiconductors and dielectrics. Energy spectrum superatom, starting with the value of the critical radius QD $a \geq a_{c}{ }^{(1)}$ (about $4 \mathrm{~nm}$ ), will consist entirely with discrete quantum levels. This is called hydrogen-superatom [2-9]. Localized above the surface of the electron is a valence QD. Quantum-discrete energy levels superatom thus, are located in the band gap matrices (dielectric or semiconductor). Electrons in superatom linked to well-defined atomic orbitals and localization in the vicinity of the nucleus (QD) [2-9]. As a core advocate QD containing in its volume semiconductors and dielectrics. Ionization energy superatomic take large values (about 3 $\mathrm{eV}$ ), which is almost three orders of magnitude higher than the binding energy of excitons in semiconductors [2-9].
As the QD radius $a$ increases so that $a \gg a_{e x}{ }^{0}$, (where

$$
a_{e x}^{0}=\frac{2 \varepsilon_{1} \varepsilon_{2}}{\varepsilon_{1}+\varepsilon_{2}} \cdot \frac{\hbar^{2}}{\mu_{0} e^{2}},
$$

two-dimensional electron Bohr radius $\left(\mu_{0}=m_{e}^{(1)} m_{h}\right.$ $/\left(m_{e}{ }^{(1)}+m_{h}\right)$ - the reduced mass of the electron-hole pairs (of spatially separated electrons and holes)), spherical QDmatrix interface transforms into a flat interface. In this artificial atom electron localized on the surface (QDmatrix) becomes two-dimensional (2D) [5-9]. In the potential energy of the Hamiltonian describing the motion of an electron in a superatom, the main contribution in this case brings the energy of the Coulomb interaction of the electron with a hole [5-9]:

$$
V_{e h}(r)=-\frac{1}{2}\left(\frac{1}{\varepsilon_{1}}+\frac{1}{\varepsilon_{2}}\right) \frac{e^{2}}{r} .
$$

(where $r$ - the distance of the electron from the center QD). Polarization interaction energy of the electron and the hole with a spherical surface interface (QD- matrix) gives a much smaller contribution to the potential energy of the Hamiltonian. In this contribution a first approximation can be neglected [5-8]. The energy spectrum of twodimensional electrons in an artificial atom Coulomb took the form [5-8]:

$$
E_{n}=-\frac{R y_{e x}^{0}}{(n+(1 / 2))^{2}}, R y_{e x}^{0}=\frac{\left(\varepsilon_{1}+\varepsilon_{2}\right)^{2}}{4 \varepsilon_{1}^{2} \varepsilon_{2}^{2}} \cdot\left(\frac{\mu_{0}}{m_{0}}\right) R y_{0},
$$

where $n=0,1,2$ - the principal quantum number of the electron, $R y_{0}=13,606 \mathrm{eV}$ - the Rydberg constant. The binding energy of the ground state of the two-dimensional electron, according to (3), is given by:

$$
E_{e x}^{0}=-4 R y_{e x}^{0} \text {. }
$$

Under the binding energy (4) of the ground state of the electron means the energy required for the decay of the bound state of an electron and a hole (in the state with $n=0$ ) in superatom.

From the dependence of the binding energy $E_{e x}(a, \varepsilon)$ of the ground state of an electron in a superatom (obtained in [5-8] by the variational method) containing ZnSe QDs of radius $a$, it follows that the bound state of an electron occur near the spherical surface interface (QD-matrix), starting with the critical QD radius $a \geq a_{c}{ }^{(1)}=3.84 \mathrm{~nm}$. Hole while moving in a volume QD, and the electron is localized on the surface of the spherical interface (QD- matrix). In this case, the energy of the Coulomb interaction $V_{e h}(r)$ (2) between the electron and the hole, and the energy of the polarization interaction of an electron and a hole with a spherical surface interface (QD-matrix) prevail over the energy of size quantization of electron and hole in the artificial atom. Thus, in [5-8] found that the occurrence of superatom has a threshold, and it is possible only from the 
QD radius $a \geq a_{c}^{(1)}=3.84 \mathrm{~nm}$.

With the growth of the radius $a$ QD scan, an increase in the binding energy of the ground state of an electron in a superatom. In the range of radii $4.0 \leq a \leq 29.8 \mathrm{~nm}$ binding energy of the ground state of an electron in a superatom significantly exceeds (in $(4,1-76,2)$ times) the value of the exciton binding energy $\tilde{E}_{e x}^{o} \approx 21.07 \mathrm{meV}$ in a single crystal of zinc selenide [5-8]. Beginning with the QD radius $a \geq a_{c}{ }^{(2)}$ $=29.8 \mathrm{~nm}$, the binding energy of the ground state of an electron in a superatom asymptotically follow the value $E_{e x}^{0}$ $=-1.5296 \mathrm{eV}$, characterizing the binding energy of the ground state of two-dimensional electrons in an artificial atom (4) [5 -8].

The effect of a significant increase in the binding energy of the ground state of an electron in a superatom mainly determined by two factors [5-8]: 1) a significant increase in the Coulomb interaction energy $\left|V_{e h}(r)\right|$ (2) electron-hole (the "dielectric enhancement"); 2) the spatial limitation on the quantization volume QD, while with increasing radius $a$ QD scan, since the QD radius $a \geq a_{c}^{(2)}=52 a_{e x}{ }_{e x}=29.8 \mathrm{~nm}$ superatom becomes two-dimensional with the binding energy of the ground state $E_{e x}^{0}(4)$, the value of which is almost two orders of magnitude greater than the exciton binding energy in a single crystal of zinc selenide.

The effect of "dielectric enhancement" due to the fact that when the DP matrix $\varepsilon_{1}$ is significantly less than the DP QD $\varepsilon_{2}$, an important role in the interaction between the electron and the hole in the superatom playing field created by these quasi-particles in the matrix. At the same time, the interaction between the electron and the hole in the superatom is much greater than in a semiconductor with DP $\varepsilon_{2}[5-8]$.

In [13] studied the optical properties of borosilicate glass samples containing QD zinc selenide. According to X-ray diffraction measurements, the mean radii $\bar{a} \mathrm{QD} \mathrm{ZnSe}$, ranged $\bar{a} \approx 2.0-4.8 \mathrm{~nm}$. At low concentrations of QDs $(x=$ $0.003 \%)$, their interaction can be neglected. The optical properties of such QN mainly determine the energy spectrum of electrons and holes localized near the surface of a spherical single QDs grown in the glass matrix. In [13] found a maximum (peak) of the spectrum of lowtemperature luminescence $\bar{E}=2.66 \mathrm{eV}$ at $T=4.5 \mathrm{~K}$, which is below the ceiling of the band gap $\left(E_{g}=2.823 \mathrm{eV}\right)$ of a single crystal of zinc selenide. Peak shift $\bar{E}$ Low temperature photoluminescence spectrum, with respect to the band gap $\mathrm{ZnSe}$ single crystal in the short wavelength region, equal $\left(\Delta \bar{E}=\bar{E}-E_{g}=-165 \mathrm{meV}\right)$.

Comparing the energy of the ground state of the electron $\left(E_{0}(a, \varepsilon)-E_{g}\right)$ in the artificial atom with the value of the peak of the luminescence spectrum $\Delta \bar{E}=-165 \mathrm{meV}$, in [5-8] was obtained by the average radius $\bar{a}_{0} \cong 4.22 \mathrm{~nm}$ QDs zinc selenide, which was in the range of the mean radii $(\bar{a} \approx 2.0$ $-4.8 \mathrm{~nm}$ ) QDs, studied under experimental conditions [13]. Thus, the proposed model we superatom allowed to interpret the mechanism of occurrence of the peak of the luminescence spectrum $\Delta \bar{E}=-165 \mathrm{meV}$ nanosystems studied in the experimental work [13].
The distance between two (or more) of artificial atoms up to a certain critical value $D_{\mathrm{c}}$ between the QD surface (eg, a smaller value of the two Bohr radii of the electron in superatom) will result in an overlap of the outer electron orbitals superatomic and the emergence of the exchange interactions and, therefore, more powerful type of bond [29,23] (compared with ties to the natural molecules). The result can be created conditions for the emergence of artificial atoms new quantum nanosystems quasimolecules or artificial molecules [4,9,23].

Specified type of bond is new, even because, despite the nature of the exchange, it can be realized at wavelengths much longer than the characteristic lengths for known natural molecules. Indeed, the value of the overlapping integral of the outer electron wave functions in the quasimolecule essential takes over distances far exceeding the characteristic length for the known natural molecule [59,23]. This enhancement effect in the quasimolecule binding energy due to the fact that the main contribution to the binding energy makes the exchange interaction energy of the electrons, the renormalized Coulomb and polarization interactions of spatially separated electrons and holes [5-9,23].

One can also assume that the above conditions for the formation of artificial molecules can be provided and influence of other factors, such as external physical fields. This assumption is evidenced by the results of $[11,12,17$, $24]$, in which the occurrence of the effective interaction between two artificial atoms over long distances under the action of the electromagnetic field has been observed experimentally

As a result, the distance between two superatomic representing QDs cadmium sulfide with localized on the QD surface valence electrons, since some critical distance $D c$ between the surfaces of the nuclei (QD) atomic orbitals of the two valence electrons overlap (in this case the value of the overlapping integral of the outer electron wave functions takes essential ) and form a bond. The result is a quasimolecule $[4.9,23]$. Such a connection is a new type of chemical bond in the quasimolecule. The total energy $E_{0}(\widetilde{D}, \tilde{a})$ the singlet ground state quasimolecule in nanoscale took the form $[4,9,23]$ :

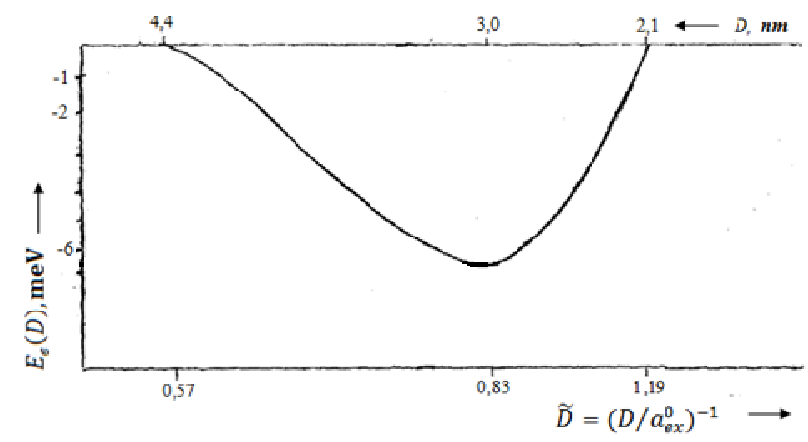

Fig 1. The dependence of the binding energy $E_{6}(a, D)$ of the singlet ground state quasi-molecule (consisting of two spherical CdS QDs with average radii of $\bar{a}_{1}=3,25 \mathrm{~nm}$ ) of the distance $D$ between the surfaces of the $Q D$. Here $a_{e x}^{0}=2.5 \mathrm{~nm}$ - the Bohr radius of an electron in a superatom. 


$$
E_{0}(\widetilde{D}, \tilde{a})=2 E_{e x}(\widetilde{a})+E_{\mathrm{B}}(\widetilde{D}, \tilde{a}),
$$

where $E_{b}(\widetilde{D}, \tilde{a})$ the binding energy of the singlet ground state of the quasimolecule, and the binding energy $E_{\text {ex }}(\tilde{a})$ of the ground state of artificial atoms (of the space separated electrons and holes) are localized on the surface of the QD found in [5-9], (parameters $\widetilde{D}=\left(D / a_{e x}^{0}\right), \tilde{a}=$ $\left.\left(a / a_{e x}^{0}\right)\right)$.

The results of the variational calculation of the binding energy $E_{b}(\widetilde{D}, \tilde{a})$ the singlet ground state of the quasimolecule in the QN, consisting of CdS QDs with average radii of $\bar{a}_{1}=3.25 \mathrm{~nm}$ (DP $\varepsilon_{2}=9.3$, the effective mass of holes in the quantum $\left.\operatorname{dot}\left(m_{h} / m_{0}\right)=5\right)$, grown in a borosilicate glass matrix (DP $\varepsilon_{1}=2$, the effective electron mass in the matrix was calculated in [25] and is equal to $\left.\left.\left(m_{e}{ }^{(1)} / m_{0}\right)=0,537\right)\right)$, which was investigated in the experimental work [13], are shown in Fig. 1 [5,6]. In [13] investigated samples borosilicate glass, doped cadmium sulphide concentrations of $x=0.003 \%$ to $1 \%$. At concentrations of QDs, since $(x \cong 0,6 \%)$, it is necessary to take into account the interaction of charge carriers localized on the QD surface.

The binding energy $E_{\beta}(\widetilde{D}, \tilde{a})$ the singlet ground state quasimolecule in the QN, comprising QDs cadmium sulfide with the mean radii $\bar{a}_{1}=3,25 \mathrm{~nm}$ has a minimum value $E_{\mathrm{B}}^{(1)}\left(D_{1}, \bar{a}_{1}\right) \approx-6,84 \mathrm{meV}$ (at a distance $D_{1} \cong 3,0 \mathrm{~nm}$ ) (see. Fig.1) ( $E_{\mathrm{B}}^{(1)}$ corresponds to the critical temperature $\left.T_{\mathrm{c}} \approx 79 \mathrm{~K}\right)[4,9,23]$. Of Fig. 1 that quasimolecule QN occurs starting from a distance $D \geq D_{c}^{(1)} \cong 2,1 \mathrm{~nm}$ between the surfaces of QDs. The formation of such quasimolecule has a threshold and is only possible in the QN, comprising QDs with average radii of $\bar{a}_{1}$, in which the distance $D$ between the surfaces of QD exceeds some critical distance $D_{c}^{(1)}[4,9,23]$.

The binding energy of $E_{e x}(\tilde{a})$ superatom $E_{\text {ex }}\left(\bar{a}_{1}\right) \cong-98$ $\mathrm{meV}$ [5-9]. The energy of the singlet ground state of the quasimolecule (5) takes the value $E_{0}\left(\widetilde{D}_{1}, \bar{a}_{1}\right) \approx$ $-202,84$ мэВ $[4,9,23]$. It should be emphasized that we used the criterion of applicability of the variational calculation of the binding energy $E_{6}(\widetilde{D}, \tilde{a})$ quasimolecule was carried out (the ratio $\left.\left(E_{\mathrm{B}}^{(1)}\left(D_{1}, \bar{a}_{1}\right) / E_{\text {ex }}\left(\bar{a}_{1}\right)\right) \cong 0,07\right)$.

With the increase of the distance $D$ between the surfaces of QD, starting with the value

$D \geq D_{c}^{(2)}=4.4 \mathrm{~nm}$, quasimolecule QN splits into two superatom (see. Fig. 1) $[4,9,23]$. Thus, quasimolecule QN may occur if conditions $D_{c}^{(1)} \leq D \leq D_{c}^{(2)}$. In addition, quasimolecule can exist only at temperatures below a certain critical temperature $T_{c}=79 \mathrm{~K}$. Biexciton (exciton quasimolecule) arose in single-crystal $\mathrm{CdS}$ with a binding energy $E_{\theta}=0.59 \mathrm{meV}$ (at a temperature of $6.84 \mathrm{~K}$ ). The binding energy $E_{b}^{(1)}$ the quasimolecule in nanosystems $E_{b}$ with exceed more than an order of magnitude [4, 9,23].

In the binding energy of the quasimolecule, as follows from the results of variational calculations $[4,9,23]$, the main contribution to the exchange interaction energy of the electrons and holes, which is substantially greater than that of giving energy of the Coulomb interaction between electrons and holes (i.e the ratio of $\leq 0,11$ ). The calculations of the binding energy of the ground state quasimolecule in the QN are variational, so they can give low values for the binding energy $\left|E_{b}(\widetilde{D}, \tilde{a})\right|$ and $\left|E_{b}^{(1)}\right|$.

Thus, the observed effect is a significant increase in the binding energy of the singlet ground state of the quasimolecule (of spatially separated electrons and holes) in the QN (more than an order of magnitude) than the binding energy of the biexciton in a single crystal of cadmium sulfide [4,9,23].

This effect is a significant increase in the binding energy of the singlet ground state of the quasimolecule due to the fact that due to the presence of the $\mathrm{QN}$ of the interface (QD-matrix), the energy of the exchange interaction between electrons and holes (the renormalized Coulomb interaction (2) spatially separated electrons and holes) in the QN, will be much greater than the energy of the exchange interaction between electrons and holes in a single crystal $[4,9,23]$.

It should be noted that in the experimental work [13], apparently, was first discovered by the interaction of the charge carriers are localized on the QD surface of cadmium sulfide. At the same time, we have proposed a model quasimolecule $[4,9,23]$ allowed to interpret the mechanism of occurrence of the peak of the absorption spectrum of nanosystems studied in [13].

In a single atom of an alkali metal valence electron moving in the Coulomb field of the atomic core [26], having the same functional dependence on $r$ as well as the Coulomb field (2), in which the valence electron in a hydrogen-like model of artificial atom [2-9]. This leads to the fact that the energy spectra of the valence electron in a single atom of an alkali metal and an artificial atom (3) describes the spectrum of a hydrogen-type [2-9, 26]. At the same time, the number of possible quantum states of the valence electron in the model of a hydrogen atom artificial is the same as the number of quantum discrete states of the valence electron in a single atom of an alkali metal [2$9,23,26]$.

In $[4,9]$, the positions of the energy levels of the valence electron in the individual atoms of alkali metals $(\mathrm{K}, \mathrm{Rb}, \mathrm{Sc})$ [26] and the new artificial atom $X$, and also the level shifts of the valence electron $\left(\Delta E_{R b}^{\mathrm{K}}, \Delta E_{S c}^{R b}, \Delta E_{\chi}^{S c}\right)$ relative to the adjacent level. In [4,9] it was assumed that the shift of the energy level $E_{x}$ artificial atom $X$ (with respect to the energy level $E_{s c}$ of the atom $\mathrm{Sc}$ ) will be the same as the shift of the energy levels $E_{R b}$ of the atom $\mathrm{Rb}$ (relative to the energy level $E_{s c}$ of the atom Sc), (i.e $\Delta E_{x}^{S c}=\Delta E_{S c}^{R b}$ ). Then, the level of the valence electron artificial atom take a value $E x$ $=-593 \mathrm{meV}$. Using the dependence of the binding energy $E_{e x}(a, \varepsilon)$ of the ground state of an electron in an artificial atom, obtained in [5,6] by the variational method, containing zinc selenide QD radius $a$, in [4,9] found the radius QD zinc selenide $a 1=5,4 \mathrm{~nm}$, which corresponded to the level $E x=-593 \mathrm{meV}$. It should be noted that the energy levels of a valence electron in the individual atoms of alkali metals (K, Rb, Sc) [26] and the new artificial atom 
$X$ is in the infrared spectral region [4,9].

Thus, we proposed in [2-9] a new model of an artificial atom allowed to offer and calculate a new nanogeterostructure quantum dot - artificial atom $X$, the natural analogue of the group of alkali metals, following the cesium and absent in the periodic Mendeleev [4,9].

Briefly discuss the differences between artificial spectroscopy [5-9] and the natural hydrogen-like atoms [26]:

1 In effect, the emergence of artificial atoms and in effect a substantial increase in the binding energy of the ground state of an artificial atom, the pivotal role played by the interface (QD - matrix). A characteristic feature of the emergence of the electronic states in such superatom (ie, the formation of superatoms) is the existence of a critical size of the nanoparticles $a \mathrm{c}$ (about $5 \mathrm{~nm}$ ). In this connection it should be noted that for substances which are capable of forming atoms should be a region of the spherical nanoparticles

$$
a_{\alpha}<a<a_{C}
$$

(where $a_{a}$ is the atomic dimensions), in which it is impossible superatomic education. Therefore, the possibility of a continuous transition from the states to the states of an ordinary atom superatomic may be carried out only under certain conditions related either to penetrate the charge carriers through the interface (nanoparticle - matrix) in the surface layer, or at atomic dimensions $a$ c (about one $\mathrm{nm}$ ) under which the above arguments inapplicable.

2 Long-distance asymptotic behavior of the potential energy of the electron $\left(r\left(^{-4)}\right)\right.$ leads to the existence of a finite number of energy levels in superatom. By varying the radius of $a$ QD can be directed superatoms receive a given number of quantum energy levels. This circumstance can be used for nanosystems containing only two levels. Such duplex nanosystems relevant as nanolasers active region and in the memory elements of optical nanocomputers.

3 Changing the radius of $a$ QD can obtain transitions between levels in the quantum-superatom with prescribed energy, located in the range from infrared to ultraviolet.

\section{Future Studies of Artificial Atoms and Molecules}

Found in superatom features local charge states due to their dependence on the binding energy of the size of nanoparticles and, in particular, the existence of a critical size of the nanoparticles, may be of interest for the development of new methods of spectroscopic control of ultradispersion media. Of particular interest is the development of such methods, for example, to control the nucleation of new phases during radiation or thermal effects on the multi-component system, especially in the extreme conditions of synthesis, etc. [2-9,27,28].

These results suggest the possibility in principle of creating a plurality of artificial atoms, including natural analogues, with new physical and chemical properties [2-9]. Based on them, we can construct new quantum nanosystems - quasimolecules and quasicrystals management symmetry and lattice period which will allow to realize the unique physical and chemical effects and phenomena, to create new principles in materials science [2-9, 23].

The observed effect is a significant increase in the binding energy of an electron in a hydrogen-superatom [5-9] (almost two orders of magnitude greater than in closed nanosystems, in which electrons and holes are localized in the volume QD) can afford to use them as the active medium of a new generation nanolasers emitting in the optical spectrum [27], as well as a base element of a new generation of optical nanocomputers $[5,6,27,28]$.

\section{Conclusions}

Scientific direction, studied physics, chemistry and technology of artificial atoms is new and is being developed as the current and future research in leading laboratories around the world. At the Chuiko Institute of Surface Chemistry of National Academy of Sciences of Ukraine first proposed and theoretically investigated a new model of an artificial atom - a nanoheterostructures of space - separated electrons and holes; developed approaches to the construction of new, unique quantum nanosystems - artificial atoms, molecules, artificial and synthetic crystals.

\section{References}

[1] Ashoori, R.C. (1996). Electrons in artificial atoms. Nature. 379 , No. 6564, 413-419.

[2] Pokutnyi, S.I., Gorbyk, P.P. (2013). Electronic properties of nanoscale quasiatomic structures. Progr. Phys. Metal. 14, No. $4,353-378$.

[3] Pokutnyi, S.I., Gorbyk, P.P. (2013). Superatoms in quasizero-dimensional nanostructures. J. Applied Chem. 1, No. 1, 44-47.

[4] Pokutnyi, S.I., Gorbyk ,P.P. (2014). Superatoms in quasiatomic nanogeterostructures: Theory. J. Applied Chem. 2, No. 4, 16 - 21 .

[5] Pokutnyi, S.I. (2013). Binding energy of the exciton of a spatially separated electron and hole in quasi-zerodimensional nanosystems. Technical Physics Letters. 39, No. $3,233-235$.

[6] Pokutnyi, S.I. (2013). On an exciton with a spatially separated electron and hole in quasi-zero-dimensional nanostructures. Semiconductors. 47, No. 6, 791-798.

[7] Pokutnyi, S.I. (2014). Theory of excitons formed from spatially separated electrons and holes in quasi- zerodimensional semiconductor nanosytems. SOP Trans. Theoretical Physics. 1, No. 2, 55- 67. 
[8] Pokutnyi, S.I. (2014). Exciton states spectroscopy in quasizero-dimensional nanosructures: Theory. Optics. Special Issue: Optics and spectroscopy of the charge carriers and exciton states in quasi-zero-dimensional nanostructures. 3, No.1, 2-9.

[9] Pokutnyi, S.I. (2014). Theory of excitons and excitonic quasimolecules formed from spatially separated electrons and holes in quasi-zero-dimensional nanosytems. Optics. Special Issue: Optics and spectroscopy of the charge carriers and exciton states in quasi-zero-dimensional nanostructures. 3, No.1, 10-21.

[10] Malygin, A.A., Smirnov, V.M. (2012). Chemical substances highly. News St.- Peterburg state. tehnic. univ. 15, 102-107.

[11] Lalumière, K., Sanders, B., Loo, B, Fedorov, A. (2013). Input-output theory for waveguide QED with an ensemble of inhomogeneous atoms. Phys. Rev. A. 88, No. 10. $43806-$ 43811.

[12] Loo, B., A. Fedorov,A., K. Lalumière, K. (2013). Photonmediated interactions between distant artificial atoms. Science. 342, No. 6165. 1494 - 1496.

[13] Bondar, N.V., Brodyn, M.S. (2010). Optical properties of semiconductor nanostructures. Physics E. 4, No. 10. 15491555 .

[14] Dzyuba, V. P., Krasnok, A.E., Kulchin, J. N. (2010). Optical properties of the dielectric nanoparticles inserted into a dielectric matrix. Technical Physics Letters. 36, No. 21. 1-9.

[15] Kulchin, J. N., Dzyuba V. P. (2010). Nonoptical properties of the dielectric nanoparticles inserted into a dielectric matrix. Pacific Science Rev. 12, No. 1. 102-105.

[16] Bulavin, L. (2009). Thermophysical properties of carbon nanotubes in toluene under high pressure. J. Molecular Liquids. 150, No. 7, 1-3.

[17] Malyukin, Y.V. (2010). Activation nanocrystals dielectric. Radiation Measurem. 4, No. 3, 589-594.
[18] Latyshev, A.N., Ovchinnikov, O.V., Smirnov, M.S. (2011). Features of the charge transfer in the interaction of molecules with sensabilizatorov nanocrystals. J. Appl. spectroscopy. 78, No.3, 481 - 484.

[19] Suvorova,T.I., Latyshev, A.N., Ovchinnikov, O.V., Smirnov, M.S. (2012). Enhancing the luminescence of dye molecules in the presence of silver nanoparticles. J. Optical Technol. 79, No.1, 79-82.

[20] Borysenko, M.V., Bogatyrev, V.M. (2004). Application of chromium-containing silica for synthesising functional glasslike materials by the sol-gel method. J. Sol-Gel Sci. Technol. 32, No. 3, 327-331.

[21] Borysenko, M.V., Sulim, I.Y., Borysenko, L.I. (2008). Modification of fumed silica atsetilatsetonanom zirconia. Theor. Eksper. Chem. 44, No.3, 191 - 195.

[22] Pokutnyi, S.I. (2007). Exciton states in semiconductor quantum dots in framework of the modified effective mass method. Semiconductors. 41, No. 11, 1323-1400.

[23] Pokutnyi S.I. (2013). Biexcitons formed from spatially separated electrons and holes in quasi-zero-dimensional nanosystems. Semiconductors. 47, No. 12, 1626-1635.

[24] Pokutnyi, S.I., Gorbyk, P.P. (2014). Quasi-zero-dimensional nanostructures: Excitonic quasimolecules. J. Applied Chem. 2, No. 1, 1-4.

[25] Pokutnyi, S.I. (1995). Exciton in quasi-zero-dimensional nanostructures. Physics Letters A. 203, No. 5,6, 388-394.

[26] Frish, S.E. (1963). Optical spectra of atoms. Nauka, Moscow.

[27] Pokutnyi, S.I. (2005). Optical nanolaser on the heavy hole transition in semiconductor nanocrystals. Physics Letters A. 342, No. 5, 347-352.

[28] Pokutnyi, S.I., Gorbyk, P.P. (2013). Absorption of light in electron states in quasi-zero-dimensional nanostructures. Optics. 2, No. 4, 47-50. 